Cambouis, la revue des sciences sociales aux mains sales

\title{
Approche des classes sociales à partir des statistiques communales
}

\section{Pierre Vergés}

\author{
CNRS-CACES
}

Résumé : Très ingénument, cette communication hasarde une réponse à la confrontation CSP/classes sociales qui nous était proposée au point B.1.1 de la grille thématique de cette table ronde. Nous étant intéressé à l'observation du changement économique et social à l'échelon communal, nous avons eu à résoudre les problèmes de passage d'une problématique conceptuelle à la description statistique sous la forte contrainte de réalité qu'est la connaissance qualitative de la commune obtenue par le chercheur à travers les entretiens, l'observation et l'enquête. Si l'on veut tenir les deux bouts de cette confrontation, il faut se refuser à un double réductionnisme. D'une part, celui de l'explication de la dynamique sociale communale par le seul jeu des classes au plan national : on donne alors à la commune un rôle illustratif et on s'interdit la prise en considération du fonctionnement local des classes sociales. D'autre part, celui de la simple modélisation statistique qui tire ses conclusions d'un ensemble de comparaisons entre la commune et d'autres communes ou entre la commune et des références plus larges (canton, département, France) : on ne définit alors que des spécificités relatives à une moyenne ou à un accroissement moyen, mais sans avoir les moyens d'expliquer ces spécificités. Il convient alors de mettre en œuvre une démarche où les deux termes sont le fruit d'élaborations méthodologiques ayant entre elles une correspondance certaine. Nous avons tout d'abord cherché des solutions générales qui soient valables quel que soit le découpage géographique; nous verrons qu'elles ne sont pas fiables. Aussi nous nous sommes orientés dans une voie qui tient compte des particularités de l'échelon communal. En dernier lieu, nous avons confronté le résultat obtenu à notre corps d'hypothèse pour en montrer la relativité et l'intérêt.

Date de publication : 28/06/2021

Dossier : Sociologie et statistique

Comment citer : $10.52983 /$ crev.vi0.53

Licence: Cambouis publie ses contenus selon les termes de la Licence Creative Commons Attribution - Pas d'Utilisation Commerciale - Pas de Modification 4.0 International. Les auteurices gardent leurs droits de propriété intellectuelle pleine et entière sur leurs articles. 


\section{Approche des classes sociales à partir des statistiques communales}

\section{Pierre Vergés}

CNRS-CACES

Très ingénument, cette communication hasarde une réponse à la confrontation CSP/classes sociales qui nous était proposée au point B.1.1 de la grille thématique de cette table ronde.

Nous étant intéressé à l'observation du changement économique et social à l'échelon communal, nous avons eu à résoudre les problèmes de passage d'une problématique conceptuelle à la description statistique sous la forte contrainte de réalité qu'est la connaissance qualitative de la commune obtenue par le chercheur à travers les entretiens, l'observation et l'enquête.

Les difficultés de cette confrontation CSP/classes sociales sont d'abord théoriques, il ne faut pas se le cacher. En effet, on nous objectera qu'il est absurde de chercher à mettre en évidence des classes sociales au niveau de la commune, car les classes sont, pour certains, un simple outil conceptuel dont le chiffrement est inintéressant, seul compte le modèle explicatif ; pour bien d'autres, la notion de classe sociale est totalement liée à la formation sociale, c'est-à-dire à l'espace national. De plus, l'observation de la structure sociale d'une commune montre le caractère tronqué de cette dernière : les patrons peuvent décider à des centaines de kilomètres de là, un groupe social peut être totalement absent. Tous ces arguments militent en faveur d'une connaissance en termes de «statistiques comparatives » de la commune (où la CSP est reine), dans une tradition empiriste ou illustrative. Il n'y a alors plus trace pour une théorie sociologique articulée à une connaissance statistique.

Si l'on veut tenir les deux bouts de cette confrontation, il faut se refuser à un double réductionnisme :

- d'une part, celui de l'explication de la dynamique sociale communale par le seul jeu des classes au plan national ; on donne alors à la commune un rôle illustratif et on s'interdit la prise en considération du fonctionnement local des classes sociales;

- d'autre part, celui de la simple modélisation statistique qui tire ses conclusions d'un ensemble de comparaisons entre la commune et d'autres communes ou entre la commune et des références plus larges (canton, département, France). On ne définit alors que des spécificités relatives à une moyenne ou à un accroissement moyen (comme dans le cas des coefficients de Dunn et Perloff), mais sans avoir les moyens d'expliquer ces spécificités. Quel rapport peut-on établir entre la plus ou moins grande spécificité relative d'une CSP et l'existence de classes 
sociales dans une localité ? Cette question reste sans réponse dans de telles problématiques.

Il convient alors de mettre en œuvre une démarche où les deux termes sont le fruit d'élaborations méthodologiques ayant entre elles une correspondance certaine.

Nous avons tout d'abord cherché des solutions générales qui soient valables quel que soit le découpage géographique; nous verrons qu'elles ne sont pas fiables. Aussi nous nous sommes orientés dans une voie qui tient compte des particularités de l'échelon communal. En dernier lieu, nous avons confronté le résultat obtenu à notre corps d'hypothèse pour en montrer la relativité et l'intérêt'.

\section{La correspondance CSP/classes sociales. À la recherche d'une solution générale}

Si l'on suit les recommandations de la notice descriptive des CSP (INSEE, 1954), il est possible de regrouper les CSP de diverses manières pour faire apparaître différents groupes sociaux représentant « approximativement une classification d'inspiration marxiste» (en toutes lettres dans le texte).

Nous avons été nous-même tenté par cette voie en impasse. La théorie des classes décrit un certain nombre d'oppositions dichotomiques (des contradictions). On peut alors leur faire correspondre plusieurs critères simples distinguant :

- les productifs des non-productifs,

— les salariés et les « à son compte»,

- dans les salariés, plusieurs niveaux de qualification afin de déterminer des strates (intellectuel/manuel ou encadrement/exécution).

On obtient un schéma de ce type :

$\begin{array}{lll} & \text { Productif } & \text { Non-Productif } \\ \text { À son compte } & \text { A } & \text { B } \\ \text { Salarié niveau 2 } & \text { C } & \text { D } \\ \text { Salarié niveau 1 } & \text { E } & \text { F }\end{array}$

Il faudrait alors pouvoir affecter de manière bi-univoque chaque CSP à chaque case. Pour certaines d'entre elles, cela ne semble pas poser de difficulté : les salariés agricoles (10) seront en E, les patrons de l'industrie et les agriculteurs (21) en A... Mais dans la plupart des cas, il y aura incertitude (où mettre les cadres administratifs (34): en C, en D) et même biais si l'on affecte les OS (63) en E, ignorant que dans une grande proportion, ils travaillent dans des entreprises du tertiaire, comme nous le verrons plus loin. Il n'y a pas de correspondance bi-univoque possible, plausible.

Alors, on peut tenter d'établir cette correspondance en reprenant l'information à la base, c'est-à-dire au niveau des bulletins individuels, en cherchant à coder les critères distinctifs déduits d'un système d'hypothèses sur les classes sociales. Nous avons participé à une telle opération qui pousse le chercheur à multiplier les critères (dans notre cas, il y en avait neuf). Chaque critère ayant deux valeurs, on peut se placer dans un treillis (ayant ici 512 éléments). On a alors une description très fractionnée de la structure sociale même si tous les éléments du treillis ne sont pas pris en compte.

On peut, pour simplifier, faire des analyses partielles comme celle de la figure 1 qui ne prend en compte que six critères.
1 Notre communication est largement redevable aux recherches effectuées par Arlette ApkarianLacout et Pierrette Vergés sur Martigues et par Jean-Claude Garnier et moi-même sur Manosque. 


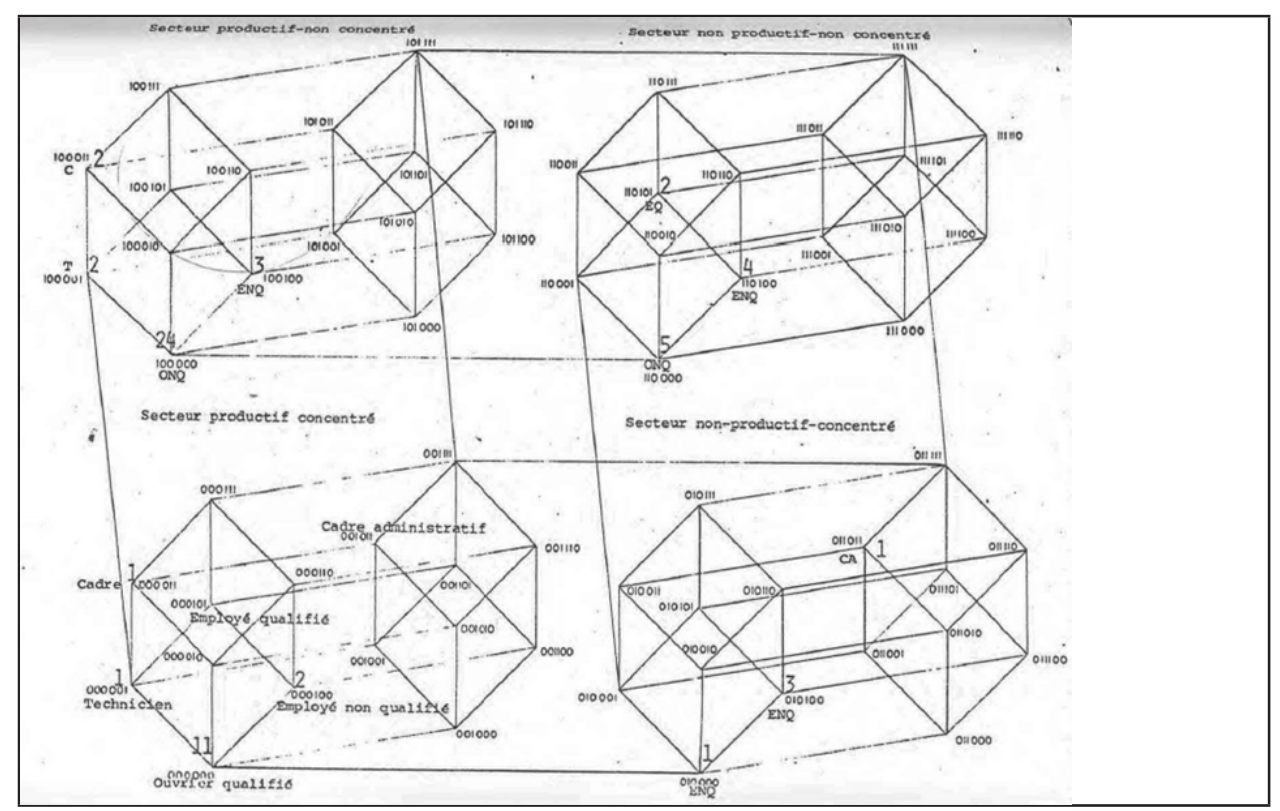

Figure 1. RGP 1975 de la commune de Vienne : actifs du secteur privé (chiffres en \% de la population active totale)

Cette méthode, par une plus grande rigueur (classification à partir de critères ayant un sens théorique), conduit à une certaine finesse de description. Mais elle ne permet pas de dépasser, finalement, cette phase descriptive. En effet, la structure mathématique du treillis d'inclusion des parties d'un ensemble n'est pas, ici, interprétable. Si, par exemple, on s'intéresse à la mobilité sociale, on observe que la distance entre employé et cadre administratif qui apparaît sur le treillis est plus grande que celle entre employé et ouvrier non qualifié, alors qu'il en est différemment dans les faits. De même, le découpage en zones contiguës que l'on peut effectuer sur le treillis pour déterminer des classes sociales est dépendant de l'ordre des éléments-bases du treillis. Il ne paraît pas possible de faire correspondre la représentation sociale des classes en termes d'opposition et/ou de proximité avec la logique d'inclusion des treillis. Il y a là une difficulté méthodologique qui limite fortement les ambitions du chercheur. Enfin, on remarquera que l'information initiale coûte cher : il faut recoder toutes les fiches individuelles du recensement.

Nous nous sommes alors orienté vers une solution qui peut paraître plus limitée au regard des rigueurs ou raideurs des approches théoriques, mais qui a l'avantage de ne se servir que des codes existants de l'Insee sans renier pour autant la définition a priori des critères de classement. D’autre part, elle peut se prévaloir de ne pas postuler une structure mathématique forte formalisant la logique dialectique des classes sociales (mathématisation tenant encore de la quadrature du cercle).

Du point de vue théorique, la position de l'acteur social dans la chaîne de production-reproduction est aussi importante que sa place dans la hiérarchie professionnelle ; aussi le croisement de l'activité par la CSP nous apparaît-il essentiel et nécessaire. Très concrètement, en prenant la nomenclature NAP pour l'activité (NAP 40) et les CSP en 30 positions (seulement les actifs), nous obtenons un tableau croisé de 120 cases dont la logique de fonctionnement n'a pas théoriquement de sens, elle n'est que le croisement de deux nomenclatures. Mais, en fait, ce tableau peut servir de base à une série d'opérations de regroupement ayant un sens relativement à nos hypothèses, car il contient l'essentiel de l'information nécessaire : le type d'activité et la place professionnelle. Certes, il nous manque une information essentielle : celle relative au capital de l'entreprise où s'exerce l'activité du salarié, mais nous verrons qu'il est possible de l'approcher à l'échelon communal. Notons aussi que la place professionnelle n'est qu'approximée par la CSP. En effet, si nous sommes 
obligés d'utiliser la CSP parce qu'elle existe pour toute la population active (alors que le code emploi ne l'est pas), il faut cependant souligner qu'avec la CSP ce que l'on obtient ne recouvre pas vraiment ce qu'indiquent les enquêtes auprès des entreprises (Enquête Structure de l'emploi) : d'un côté, c'est l'entreprise qui code, de l'autre, c'est le salarié, il y a là toute la différence entre une classification fonctionnelle et une classification en termes de représentation du poste de travail.

Ceci étant posé, il faut s'attacher à définir les critères de classements qui nous permettent de regrouper les codes NAP et CSP. Dans le code NAP, on utilise deux critères qui se croisent.

- D'une part, à quelle logique répond la marche de l'entreprise : celle de l'État ou celle du capital ? Est-ce une entreprise devant dégager un profit (même nationalisé) ou un service public?

- D'autre part, l'activité fait-elle partie du secteur productif ou du secteur des services et de la transmission des produits? Ici, notons qu'il est difficile de passer outre, malgré toutes les discussions théoriques sur « productif - non productif », à la coupure secondaire/tertiaire qui organise le code NAP (TOI à T24, d'un côté, et T25 à T38, de l'autre).

Ainsi, on repérera quatre groupes : un service d'État orienté soit vers la production, soit vers la collectivité, et des établissements soit dans le secteur de la production soit dans celui des services et de la transmission des produits. Faire une correspondance bi-univoque entre les activités du code NAP et ces groupes à un échelon autre que celui de la commune paraît possible, mais comportera quelques zones d'incertitude.

Le regroupement des CSP est à la fois plus général et plus contraint par la nature des éléments qui entrent dans la composition de ce code. Ainsi, il n'est pas possible de tenir compte d'un critère du type manuel/intellectuel, ou en position hiérarchique/ou non. Cependant, on peut,

_ d'une part, utiliser un critère simple : à son compte/salarié pour approximer la relation salariale,

- d'autre part, établir une série de strates chez les salariés distinguant de trois à six niveaux, par exemple : les personnes non qualifiées (OS, personnel de service...), les qualifiés (OQ, employés...), ceux qui ont un pouvoir technique ou hiérarchique (technicien, contremaître, cadre moyen...), enfin les cadres supérieurs (ingénieur, professeur...). Cette stratification repose sur l'opposition exécution/pouvoir et sur l'existence de deux natures de maîtrise de ces éléments ; mais on peut toujours démontrer le caractère relatif de son application aux CSP.

Toute élaboration méthodologique de données a un certain caractère ad hoc. Le nôtre tient compte de la nature des informations existantes (Insee, recensement général de la population) et de la finesse nécessaire et suffisante pour pouvoir parler de classes sociales à l'échelon communal. Aussi critiquable soit-il, il nous a paru assez adapté et signifiant, même au regard de notre système d'hypothèses.

\section{Les problèmes posés par la localisation communale des études}

Notre parti pris d'effectuer une étude localisée allie difficulté et commodité. Difficulté parce que la précision que le chercheur obtient de ses enquêtes sur le terrain n'a pas toujours sa correspondance dans les sources statistiques. Commodité, car l'existence de phénomènes massifs, tel celui d'entreprises ou de branches d'activité dominantes, et les informations que le chercheur 
recueille par ailleurs lui permettent de donner une signification précise à des codes généraux regroupant souvent des réalités fort différentes.

Lorsque le chercheur en sciences sociales se donne pour terrain d'enquête une commune, l'arsenal statistique qu'il trouve auprès de l'Insee ou des différents ministères est assez réduit. Ainsi, la connaissance statistique qu'il peut avoir de l'emploi est un peu douteuse : certains fichiers ont trop d'entreprises, d'autres au contraire ignorent des pans entiers de l'économie. Le recensement est bien sûr la source la plus utilisée, mais il n'est pas toujours récent ou exhaustif. Or, les problèmes de fiabilité sont encore plus aigus à l'échelon communal qu'ailleurs, car on fera sourire les habitants si on leur cite des chiffres par trop différents de ceux dont ils ont une appréciation directe (le nombre de médecins, le personnel de telle entreprise ou de tel service...). De même, la disparition ou l'apparition d'une entreprise est immédiatement perçue alors que son enregistrement demande un certain délai. À l'échelon d'une ville petite ou moyenne, ces difficultés sont majeures et demandent une enquête complémentaire sur le terrain auprès des différents partenaires sociaux.

Cette enquête a d'ailleurs plus d'intérêt que le seul infléchissement des statistiques officielles ; elle nous permet de complexifier notre premier schéma de classement du tableau NAP-CSP. Nous avions remarqué (au point 1) l'absence de prise en considération de la nature du capital des entreprises. À l'échelle de la commune, cette absence est une véritable carence, car le rapport que les entreprises entretiennent avec la localité est en relation étroite avec cette nature du capital. Or, ce rapport est au centre de la dynamique de l'emploi de toute commune.

Pour formaliser ce rapport, on peut distinguer trois types d'entreprises en fonction de leur espace économique, et donc de la place de la commune dans cet espace.

- Pour les premiers établissements, ceux dont le capital est « financier» et dont l'espace économique est national, voire international, on peut dire que les intérêts économiques locaux ne sont que des paramètres dans une stratégie globale du groupe ou du secteur économique auxquels ils appartiennent.

- Les seconds ont un espace économique dépassant la localité en bien des points (marché, recrutement du personnel, plusieurs établissements...). Mais l'économie locale et l'environnement industriel sont le cadre déterminant de leur fonctionnement (sous-traitance, capital local...).

- Les derniers ont une activité économique pratiquement totalement déterminée par la localité (capital, marché) ou par les fonctions de cette dernière (fonction de pôle de service, de tourisme...).

Cette classification est essentielle à notre propos, car ces types d'entreprises ont des modes de gestion du personnel fort différents, elles développent des rapports sociaux de production spécifiques et elles donnent à leur personnel une place particulière dans la localité (par le recrutement, par l'identité d'entreprise, par le rapport à l'histoire locale...). On repère ici un fractionnement économique des classes sociales dont nous avons pu mesurer la pertinence. L'ouvrier protégé d'une grande entreprise ayant une position unique dans la localité n'a pas les mêmes comportements que son collègue de même qualification qui se trouve dans une petite entreprise sous-traitante dont le patron, ancien ou actuel notable, développe des relations paternalistes.

Avec l'introduction de cette nouvelle variable, on possède maintenant un tableau comprenant 35 cases (voir figure 2) qui définit un ensemble de groupes d'actifs fractionnés par des déterminants économiques. Ce tableau est bien sûr, en cet état, uniquement descriptif. Il permet la mise en évidence de groupes ayant un poids suffisant pour peser sur la localité. L'évolution dans le 
temps d'un tel tableau montrerait l'effacement quantitatif de certains groupes ayant eu des positions hégémoniques.

Mais peut-on parler déjà de fraction de classe? Non, car nous n'avons pas pris en considération les éléments politiques et idéologiques de la structure sociale locale. Même si cette dimension n'intéresse pas directement le propos de cette table ronde, il convient cependant d'en dire un mot, car elle situe la formalisation que nous venons de présenter.

\section{Des groupes d'actifs aux classes sociales, un dépassement des seuls critères écono- miques}

Notre perspective de découpage social, ici présenté par un tableau comportant un certain nombre de cases, exprime l'hypothèse d'un fractionnement des classes sociales par le fonctionnement du palier économique de la société.

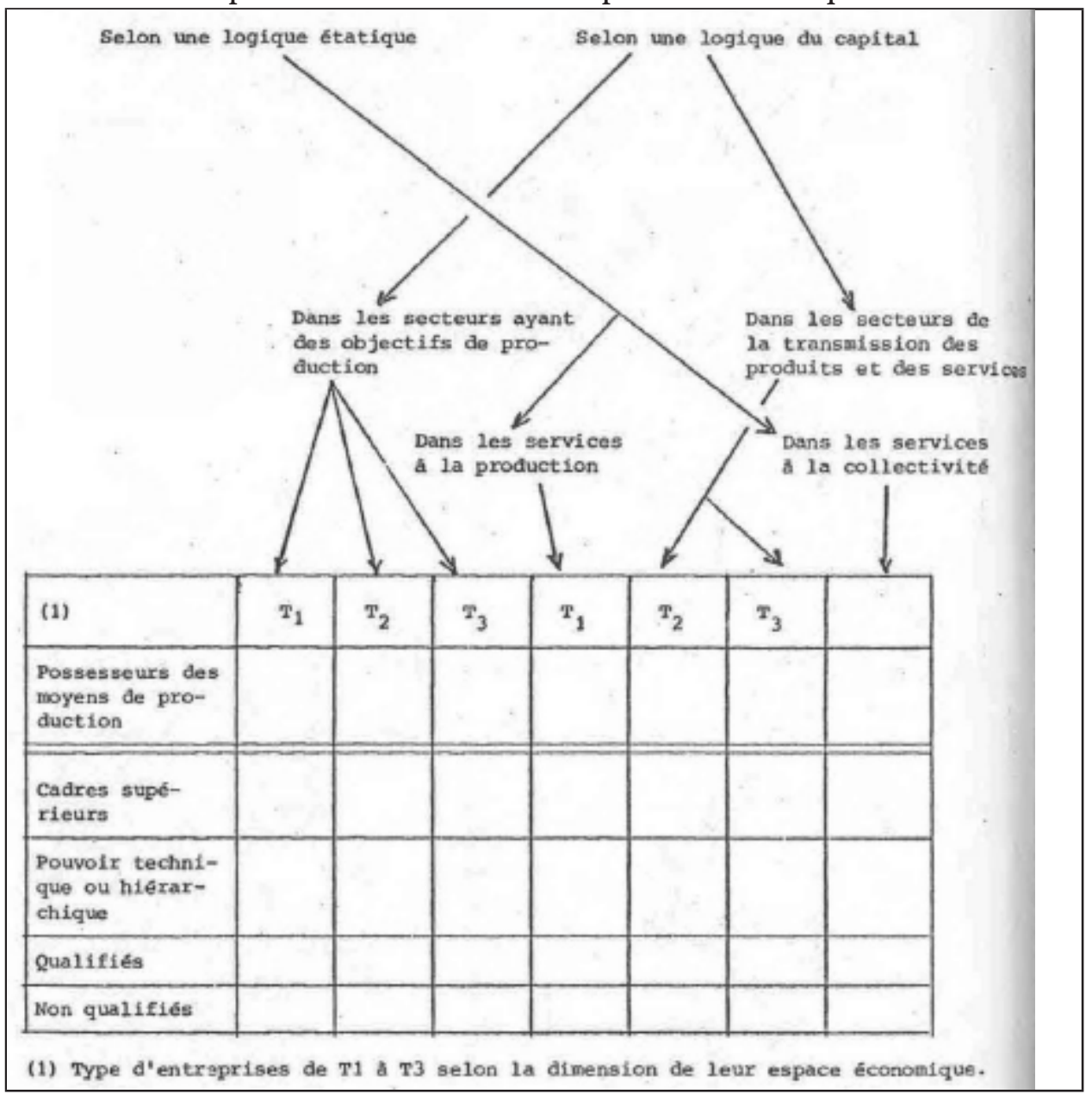

Figure 2. Tableau de croisement NAP/CSP

En effet, ce découpage n'est pas analytique : découper pour mieux comprendre et synthétiser. Il est inscrit dans le double mouvement de fractionnement/ recomposition qui anime les classes sociales. Il y a bien un fractionnement lié à la complexité du système économique. Mais il existe aussi un processus de recomposition, pour partie économique et aussi politique et idéologique, qui permet de le dépasser. L'action d'un syndicat, d'un parti n'est-il pas fondamentalement celui de réunir, regrouper, unifier des groupes sociaux aux intérêts sécants, mais non confondus? C'est à travers un tel travail que se constituent les classes. Nous approchons ce processus en mettant au jour un fractionnement initial (les groupes d'actifs) et en recherchant les regroupements qui 
s'effectuent à propos d'enjeux sociaux. On observe qu'ils s'effectuent autour de groupes ayant un rôle hégémonique. Il n'est pas rare de constater que leur place économique et même quantitative est importante. On remarque aussi que les contiguïtés sur le tableau ne sont pas sans rapport avec les chances de regroupement au sein d'une même classe. À l'échelon communal, il est intéressant de repérer que le clivage des classes ne va pas passer aux mêmes endroits du tableau selon les communes, c'est-à-dire selon l'histoire politique locale, les cultures, les groupes quantitativement dominants de la localité.

$\mathrm{Au}$ terme de ce travail, il nous semble que l'inscription d'un tableau quantitatif, à base de données Insee, dans un ensemble de données relatives à d'autres dimensions (qu'économique ou démographique), permet seule de parler de classes sociales. L'obtention systématique au plan local d'un tableau de base NAP-CSP nous paraît un service essentiel que l'Insee pourrait apporter à la connaissance statistique des classes. Les résultats que nous avons obtenus sur des villes moyennes nous font espérer maintenant un retour sur la théorie, car ils montrent l'importance du fonctionnement local des classes pour définir leur nature et leur réalité. 
InSEE, 1954, Code des CSP, Paris, Insee, $3^{\mathrm{e}}$ édition. 